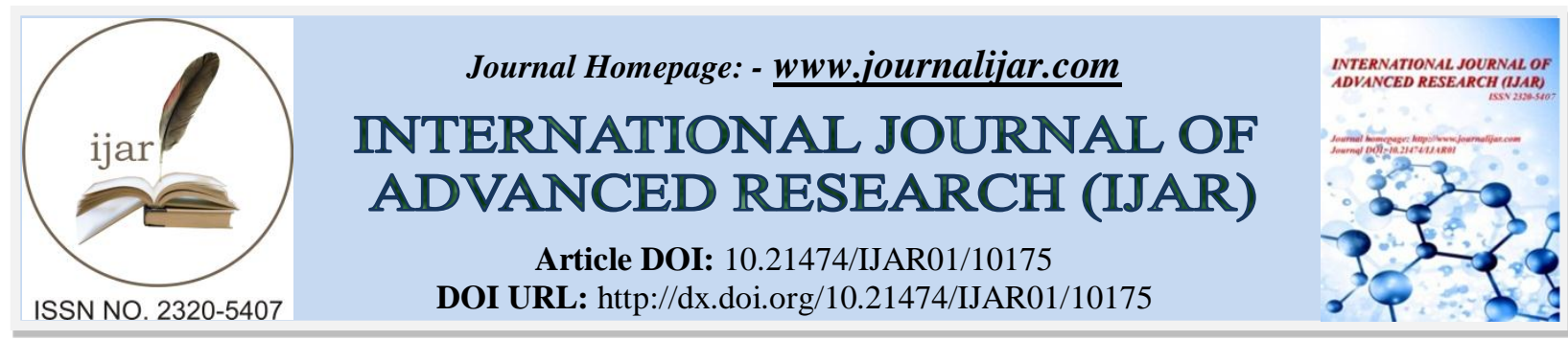

RESEARCH ARTICLE

\title{
THE ROLE OF SURFACTANTS IN LIQUID SOAPS AND ITS ANTIMICROBIAL PROPERTIES
}

\author{
S. Nazdrajic ${ }^{1}$ and A. Bratovcic ${ }^{2}$ \\ 1. Senior Assistant, Faculty of Education, University "Dzemal Bijedic" in Mostar, University Campus bb, 88000 \\ Mostar, Bosnia and Herzegovina. \\ 2. Associate Professor, Department of Physical Chemistry and Electrochemistry, Faculty of Technology, \\ University of Tuzla, Univerzitetska 8, 75000 Tuzla, Bosnia and Herzegovina.
}

\section{Manuscript Info}

\section{Manuscript History}

Received: 05 October 2019

Final Accepted: 07 November 2019

Published: December 2019

Key words:-

Liquid soap, Surfactants, Antimicrobial properties

\begin{abstract}
A typical liquid soap is composed of a mixture of different types of surfactants to achieve the desired cleaning and foaming properties. Several thermodynamic and transport properties of surfactant solutions are affected by the size and shape of micelles. Only surfactant monomers contribute to lowering the surface and interfacial tension, while wetting and foaming properties are governed by the concentration of free monomers in solution. The success of surfactants formulation in a liquid soap is reflected on physical stability of the final product, mildness, wetting, foam volume and foam stability. Furthermore, this study reveals that when amphoteric and nonionic surfactants are used with anionic surfactants, foaming of the formulations are improved. Amphoteric surfactants have antibacterial properties, low toxicity, excellent resistance to hard water, and is suitable with various kinds of surfactants. Mixing anionic and cationic surfactants is not recommended. The aim of this paper is to show the influence of individual surfactants and their mixtures on the final properties of liquid soaps as well as the antimicrobial activity of surfactants and the advantages of biosurfactants. The highest antimicrobial activity is reported for surfactants containing 10-14 carbon atoms in their chain. The toxicity of cationic surfactants is the highest, then follow anionic and the last one is nonionic. Biosurfactants show the same functionality as the chemical synthetized surfactants, but biosurfactants are more environmentally friendly, with lower toxicity and biodegradable ability.
\end{abstract}

Copy Right, IJAR, 2019,. All rights reserved.

\section{Introduction:-}

One of the most important and extensively investigated segments of skincare market is soaps and liquid soaps. Soap is considered the oldest cosmetic product and also plays an important role in daily life. The initial development of liquid soaps may be dated to as early as the 1940s. In the 1960s and 1970s liquid soaps started to appear as institutional and hospital health care hand washing products, some using simple liquid fatty acid coco soaps and some using blends of synthetic surfactants. In the late 1970s liquid soaps were developed and launched on the global market in the U.S. The liquid soaps offered some advantages over conventional bar soaps, and they soon gained consumer acceptance and became increasingly popular. Soaps are detergents based on synthetic surfactants and

Corresponding Author:- S. Nazdrajic

Address:- Senior Assistant, Faculty of Education, University "Dzemal Bijedic" in Mostar, University 501

Campus bb, 88000 Mostar, Bosnia and Herzegovina. 
auxiliary components. Surfactants can be classified as organic compounds with enormous applications in the areas of soaps, detergents, emulsions, paints, organic synthesis, petroleum recovery, lubricants and as membrane mimics. The applications of surfactants are also useful in chemistry and chemical engineering, thermodynamics and catalysis evaluations (Chidi and Adebayo, 2018).

Surfactants are important ingredients of personal care products and household products. They are designed to remove dirty, sweat and fat from the skin and other surfaces. Surfactants are widespread used in everyday life because of their excellent performances like wetting and emulsifying (Yuan et al., 2014).

\section{Surfactants and surface activity}

Surfactants are a unique group of organic compounds containing both a hydrophobic long-chain (tail) and a hydrophilic polar group (head) in their structures. Hydrophilic surfactant head groups are organised in the shell of micelles towards water, while hydrophobic tails packed together in core try to minimise the contact with water by being in oil. When the concentration of surfactants reaches a specific narrow range, called critical micelle concentration (CMC), the molecules tend to self-associate with tails aggregating inward into an assembled micellar structure which is favoured thermodynamically in polar solvents. (Lavkush Bhaisare et al., 2015). Therefore, the critical micelle concentration of a surfactant is the concentration at which the surface concentration of surfactant molecules reaches a level where they begin to self-aggregate to form micellar structures (Nguyen et al., 2015).

The surfactant classification depends on water dissociation and the structure of hydrophilic group. The water-soluble surfactants, may be divided into two major groups: ionic surfactants and nonionic surfactants. Ionic surfactants can be divided into anionic surfactants, cationic surfactants and amphoteric surfactant. Anionic surfactant is a surfactant that carries an ionic group in the form of either a negative or a positive head; or it may carry both simultaneously in one molecule (zwitterions) (M. Toofan \& J. Toofan, 2015). More detail classification of surfactants is already described in our previous published scientific paper (Bratovcic et al., 2018).

Adsorption is the concentration of surfactants at interface, while self-assembly is the aggregation of surfactants into structure micelle. Just above the critical micelle concentration, spherical micelles represent the dominant form of self-assembly (Anachkov et al., 2018).

Contrary to micelles in water, the structure of micelles formed in non-aqueous solvents is absolutely inverse, where head groups are in polar core and hydrophobic tails in the shell. (Nguyen, 2015).

Micellization phenomena have been of great interest to both the academic community and industry. It is involved in several applications and processes. The self-assembly of amphiphiles into micelles may be studied by monitoring changes in the bulk properties of an amphiphile dispersion (e.g. conductivity, surface tension, enthalpy or the polarity around a hydrophobic fluorophore).

Micelles are spherical nanometre sized objects with a hydrophobic core and hydrophilic surface and micellization is affected by the chemistry of the amphiphile and environmental conditions such as temperature or the present ions (Odunze et al., 2019). The shape and size of micelles are governed by geometric and energetic aspects, the nature, and the concentration of surfactant, the solvent and other species in solution. The formed micelles can be cylindrical, lamellar or spherical, while their radius varies in a range from $10 \mathrm{~nm}$ to over $100 \mathrm{~nm}$. Nonionic surfactants have very low values of CMC and their micelles are larger than those of ionic surfactants. Several thermodynamic and transport properties of surfactant solutions are affected by the size and shape of micelles (Santos et al., 2016).

The dual characteristics of surfactant molecules give them a wide range of properties connected to adsorption at interfaces and self-assembly in bulk solution. Surface tension arises from an imbalance of attractive intermolecular interactions at a liquid surface. This imbalance of intermolecular attractive interactions creates excess energy at the surface compared to the bulk (the surface free energy), which forces liquid surfaces to contract, reducing the exposed surface area. When present at low concentrations, surfactant adsorption to the air-water interface is a spontaneous process resulting in an oriented monolayer, which alters the surface free energy (Czajka et al., 2015).

\section{Effect of surfactants on physicochemical properties of liquid soaps}

Physiochemical analysis is a procedure investigating physiochemical systems which made possible a determination of the nature interaction between the component of a system through the study of systems physical properties and composition (Hassan et al., 2017). The physicochemical properties of soaps determine their quality and cleansing efficacy. The key physical properties of liquid soaps can be defined and characterized by rheology, $\mathrm{pH}$ and colour. 
Normally human healthy skin has $\mathrm{pH}$ ranging from 5.4- 5.9 and a normal bacterial flora. The $\mathrm{pH}$ of liquid hand soap formulations is sometimes higher than skin $\mathrm{pH}$ because of technical requirements for viscosity and type of surfactants used.

Viscosity gives the flow characteristics of the detergent, but also has a very big influence on customer attraction (Azeez \& Abegunde, 2016). Viscosity is a liquids resistance to flow. When the intermolecular forces of attraction are strong with in a liquid there is a larger viscosity. The viscosity of liquid hand soap products is generally in the range 3000 to 5000 centipoise $(\mathrm{cP})$. If the concentration of the zwitterionic surfactant is too high, the high shear viscosity of the liquid detergent composition will increase to an exceedingly high level. Cocamidopropylbetain supported by sodium chloride could increase viscosity of detergent without need to add thickening agent (ElShattory et al., 2018). When cationic surfactants are mixed with nonionic surfactants, there is a generally need to use thickeners to increase the viscosity to a level that is expected by consumers for a cleansing composition, such as a liquid hand soap or body wash.

The mixed solutions of the anionic surfactant sodium laurylether sulfate (SLES) and the zwitterionic cocoamidopropyl betaine (CAPB) contain spherical and/or rod-like micelles, depending on the ratio and the total concentration of the two surfactants. These solutions possess nearly Newtonian behaviour and low viscosity. The addition of co-surfactants in low concentrations may significantly increase the viscosity of these solutions and even transform them into viscoelastic gels (Mitrinova et al., 2018). Bratovcic et al., 2018 investigated the influence of the type of surfactant on physicochemical properties of liquid soap. The studies have shown that by increasing concentrations of zwitterionic (amphoteric) surfactant, and by decreasing concentration of nonionic surfactant, a mild decrease in $\mathrm{pH}$ value and viscosity increase occurred.

Stability of a detergent formulation is paramount, because any change implies poor quality and negatively impact consumers' acceptance. The formulation success of a liquid soap depends on the physical stability of the product where no visible phase separation after storage occurred. Maurad et al., 2017 showed that the methyl ester sulphonate (MES) as anionic surfactant could be potentially formulated into liquid detergents and give better performance than linear alkyl benzene sulphonates (LAS) based liquid detergent. The formulated MES based products were stable for a long period, even at elevated temperature $\left(45^{\circ} \mathrm{C}\right)$.

\section{Foaming properties and stability}

Liquid soaps are products that are gaining wide appreciation by the consumer due to the best fixing of perfume and attributes such as hydration and smoothness to the skin. These products show very similar composition of shampoos, with only minor differences, for example, the amount of foam and smoothness. These characteristics are highly valued because it makes the active content, and conditioning agents which are added in higher concentration. Liquid soaps can be applied to body for facial and hand hygiene. The formulation of liquid soaps is generally constituted by surfactants, for example, lauryl ether sodium sulphate and cocamide propyl betaine, which are used in order to ensure the stability of the foam and increase the viscosity of liquid soaps (Mendes Cangussú et al., 2016).

As a mixture of different types of surfactants (anionic, cationic, nonionic and amphoteric), the formulation often shows a synergistic effect in those products, and it is the mixture that is used rather than individual surfactants. Mixtures of anionic and nonionic surfactants can provide overall benefits and advantages such as mildness, wetting, foam volume and foam stability of a formulation. Betaines and amine oxides provide performance enhancement in a mixture with anionic surfactants, mostly in foam boosting, stability, and grease removal efficiency (Blagojevic S. N. et al., 2016). Blagojevic S. M. et al., 2017 reported that a nonionic surfactant of amine oxide type significantly improves the performance of anionic/amphoteric mixed micelle systems, and because of that anionic/amphoteric/nonionic mixture may be used in considerably lower concentrations as a cleaning formulation (Blagojevic S. M. et al., 2017).

The relationship between surfactant structure and foam creation and sustainability is complex. It is widely understood that most surfactants with low CMCs foam well. Foam stability may be affected by many factors, such as concentration of surfactants, diffusion of gas between bubbles, surface viscosity, bulk viscosity, surface elasticity, and the existence and thickness of the electrical double layer (Osei-Bonsu et al., 2015). Nonionic surfactants generally foam less well as they have difficulty with lateral packing at the air/water interface. Generally, very pure surfactants, even if they have the ideal structure, do not produce good foams. The best foams are produced when blends of surfactants are used and when foam boosters are introduced (Cornwel, 2018). Ionic surfactants generally 
present better foam stability compared to nonionic surfactants, which should be attributed to electrostatic interaction forces existing in ionic surfactant solutions (Sheng et al., 2016).

\section{Effect of surfactant on antimicrobial properties of liquid soap}

Microbiological contamination is one of the wildly investigated subjects in the literature. Antimicrobial activity depends not only on activity individual ingredients but also on interactions between components, especially since that sulfobetaine individual exhibit good anti-candida activity. Literature data dealing with the antimicrobial activity of commercial liquid soaps shows that the disinfectant ability of composed products is high, however higher against gram-positive bacteria (Wieczorek et al., 2017).

Cationic surfactants are recognized as molecules demonstrating antimicrobial action. Literature data indicate that antibacterial activity strongly depends on the structure of surfactants. Zhi et al., 2014 described antimicrobial activity of saccharide-amide cationic surfactants comprising hydroxyethyl group and a sugar moiety $\mathrm{N}$-methyl-Nhydroxyethyl group-N [3-(gluconamide)-propyl]-N-alkylammonium bromide diversified in terms of chain length (10, 12 and 14 carbon atoms). Authors stated that all obtained surfactants demonstrated antibacterial activity against Escherichia coli and Staphylococcus aureus, however the biological efficiency was connected with the chain length. Surfactant with 12 carbon atoms had the best antibacterial activity, while compound with 10 carbon atoms had the worst. Many authors reported that the highest antimicrobial activity is observed, when surfactants contain $10-14$ carbon atoms in their chain (Kosmowska et al., 2014).

\section{Antimicrobial test activity}

The standard antimicrobial test activity of liquid soaps is well diffusion assay. This test means application of bacterial or yeast cultures to a sterile Petri dish with appropriate agar medium. Next the wells (10 $\mathrm{mm}$ of diameter) are created. In addition, certain amount of aqueous solution of liquid soaps solutions is poured into each well. Wells were made in seed agar and the test samples (50\% aqueous solution) are introduced directly into these wells. After incubation (at $30^{\circ} \mathrm{C}$ or $37^{\circ} \mathrm{C}$ for $48 \mathrm{~h}$ ), the distances between the edge of the well and the end of the clear zones around the wells are measured (Gwiazdowska \& Wieczorek, 2014).

\section{Toxicity of surfactants}

Surfactant analytics are very important both for the development of new surfactants and for their production, application and their safety in the environment. Generally, nonionic surfactants are less toxic than ionic surfactants, their toxicity increases with increasing hydrophobic chain length and reduce with increasing ethoxylate chain length. The toxicity of cationic surfactants is the biggest, and the toxicity of anionic surfactants is between that of nonionic surfactants and cationic surfactants. There have been the reports that sodium dodecyl benzene sulfonate (SDBS) is absorbed through the skin, they damage the liver and cause other chronic symptoms, as well as teratogenic and carcinogenic diseases (Yuan et al., 2014).

It has been found that active ingredients, despite being the number one in virtually all soaps, shampoos and cleansers, cause skin irritation. Clinical studies of Sodium Lauryl Sulfate (SLS) as a skin irritant has shown that skin irritations occur at concentrations of $0.5 \%$, which is $1 / 60$ th the concentration found in some hand soaps (Ara et al., 2018).

There is a growing interest in the study of the environmental properties of surfactant mixtures since in some cases antagonistic or synergistic toxic effects have been shown for some mixtures. For example, it is found lower toxicity in binary mixtures between sodium linear alkylbenzene sulfonate, sodium dodecyl sulfate, sodium oleate, and alcohol ethoxylates (Ríos et al., 2018).

Fernández-Serrano et al., 2014 studied the interaction between different types of surfactants which are often used as co-surfactants in detergent formulations to establish the best way to determine which surfactants will work better together. The study of anionic and nonionic surfactants is particularly interesting. In order to establish synergisms between the different surfactants, Toxicity Unit values (TU) were calculated as the ratio between the measured EC50 value and the average of EC50 values for individual surfactants. In this case, binary mixtures measurements indicated that the least toxic mixture was formed by the surfactant having lower individual toxicity. It was also demonstrated antagonism for the toxicity of some binary mixtures of ether carboxylic derivative and amine-oxide based surfactants, TU $<1$ (Fernández-Serrano et al., 2014). 
Barmentlo et al., 2015 showed that for some surfactants within a specific surfactant group (homologues sharing the same head group), toxicity is observed to increase with increasing alkyl chain length due to increased hydrophobicity (Barmentlo et al., 2015).

Gemini alkylammonium surfactants show biological and surface activities much higher than monomeric analogues (Tehrani-Bagha et al., 2015). It means that the same biocidal or surface effect can be reached using much smaller amounts of gemini surfactants, what has a fundamental importance from a toxicological and ecological point of view. Garcia et al., 2016 investigated biodegradability and aquatic toxicity of quaternary ammonium-based gemini surfactants and found that these surfactants are less toxic to the aquatic life than the monomeric ones. Furthermore, the aquatic toxicity can be reduced by increasing the hydrophilicity of the surfactant molecule by including a heteroatom in the spacer or replacing a methyl by a hydroxyethyl group in the ammonium polar head group (Garcia et al., 2016).

\section{Biosurfactants in soaps}

The environmental concerns about the effect of toxic and conventional surfactants have increased the demand for renewable and biodegradable surfactants with low toxicity and other useful effects. Biosurfactants can be defined as the surface-active biomolecules produced by microorganisms with wide-range of applications (Vijayakumar \& Saravanan, 2015). Biosurfactants have the same functionality as the chemical synthesized surfactants, such as lowering the surface and interfacial tension, emulsification, solubilization, foaming, dispersion, wetting, antimicrobial activity, cleansing and many other useful properties (Bhattacharya et al., 2017).

Indeed, the performance of the biosurfactants compares favourably with that of chemical surfactants in detergent mixtures (Mortensen et al., 2017). Akbari et al., 2018 found that the nature of biosurfactants makes them a promising molecule due to their availability, biodegradability, low toxicity, and renewability. Therefore, these compounds can be used in food, cosmetic, medicine and many other products related directly to human's safety (Akbari et al., 2018). Study by Otzen, 2017 showed that addition of a cyclic lipopeptide improved washing performance of different detergent mixtures already containing different commercial surfactants. In study performed by Bouassida et al., 2018 the biosurfactant removed $81 \%$ of engine oil, while the commercial detergent removed $34 \%$. In this same study was observed that the chemical surfactant isolated exhibited less cleaning efficiency of oil (62\%) when compared to the commercial Bacillus subtilis SPB1 biosurfactant (75\%) (Bouassida et al., 2018).

\section{Results and Discussion:-}

Amphoteric surfactants generally are rarely used alone and are often used with fatty alcohol sulfates. This combination gives rise to improve the solubility, increase the viscosity and the foam stability reduce and the irritation property. Lauryl and lauryl ether sulfates are most commonly found in skin and hair detergents, which are anionic surfactants. These surfactants have a more irritating effect on the skin and eyes than nonionic and amphoteric and, due to the nature of the alkyl chain, greatly affect the irritation potential. Addition of ethoxylate to alkyl sulfates reduces the reactivity of the skin and reduces the effect of foam formation, which is the main reason for using alkyl ether sulfate. The most widely used and for washing are the most important anionic surfactants, especially alkylbenzenesulfonates $\left(\mathrm{R}-\mathrm{C}_{6} \mathrm{H}_{4}-\mathrm{SO}_{3} \mathrm{H}\right.$; $\mathrm{R}$ is a hydrocarbon group), alkylsulfonates $\left(\mathrm{R}-\mathrm{SO}_{3} \mathrm{H}\right)$ and alkylsulfates $\left(\mathrm{RO}-\mathrm{SO}_{3} \mathrm{H}\right)$. Mixtures of anionic and nonionic surfactants show specific behavior because of significant reduction in the mixture CMC. It is due to the fact that in mixed micelles, the polar group of the ionic surfactant is located further than the polar group in micelles of a pure ionic surfactant, and, therefore, the repulsion energy between the charged groups is smaller. Cationic agents, positively charged, have lower foaming properties than anionic surfactants. Because of their considerable bactericidal activity against a wide range of microorganisms, they may be used as antimicrobial preservatives rather than as surfactants. Cationic surfactants prove to be at least equally irritating, but more cytotoxic than anionics.

\section{Conclusions:-}

The soaps are cleaning agents routinely used for cleaning purposes and removing germs. Surfactants as major components of liquid soaps are widely used in everyday life to remove dirty and fats from skin and other surfaces.

The success of surfactants formulation in a liquid soap is reflected on physical stability of the final product, mildness, wetting, foam volume and foam stability. Both amphoteric and nonionic surfactants provide performance enhancements in a mixture with anionic surfactants, mostly in foam boosting, stability and grease removal 
efficiency. Most surfactants with lower CMC foam better, but foam creation depend on surfactant structure, concentration, diffusion of gas between bubbles, surface viscosity and elasticity and thickness of the electrical double layer. Cationic surfactants are recognized as molecules with antimicrobial action, while the highest antimicrobial activity is reported for surfactants which contain 10-14 carbon atoms in their chain. The toxicity of cationic surfactants is the highest, then follow anionic and the last one is nonionic. The toxicity of nonionic surfactants increases by increasing hydrophobic chain length and reduce by increasing ethoxylate chain length. Biosurfactants show the same functionality as the chemical synthetized surfactants, but biosurfactants are more environmentally friendly, with lower toxicity and biodegradable ability.

\section{References:-}

1. Akbari S., Nour A. H., Yunus R. M., Farhan A. H. (2018). Biosurfactants as promising multifunctional agent: A mini review, International Journal of Innovative Research and Scientific Studies, 1 (1):1-6.

2. Anachkov S. E., Georgieva G. S., Abezgauz L., Danino D., Kralchevsky P. A. (2018). Viscosity Peak due to Shape Transition from Wormlike to Disklike Micelles: Effect of Dodecanoic Acid, Langmuir, 34 (16), 4897 4907.

3. Ara T., Laway G. N., Deva A. S., Deva B., Bhatia N., Khan R. A. (2018). Hazardous effects of sodium lauryl sulfate and sodium laureth sulfate, An overview, World Journal of Pharmacy and Pharmaceutical Sciences, 7(5): 282-292.

4. Azeez O. S. \& Abegunde G. S. (2016). Production and characterization of liquid detergents from some agricultural waste products, Nigerian Journal of Technology, 35, (1) $60-65$.

5. Barmentlo SH, Stel JM, van Doorn M. (2015). Acute and chronic toxicity of short chained perfluoroalkyl substances to Daphnia magna, Environ Pollut., 198: 47-53.

6. Bhattacharya B., Ghosh T.K., Das N. (2017). Application of Bio-Surfactants in Cosmetics and Pharmaceutical Industry, Sch. Acad. J. Pharm. 6, 149-157.

7. Blagojevic S. N., Blagojevic S. M., Pejic N. D. (2016). Performance and Efficiency of Anionic Dishwashing Liquids with Amphoteric and Nonionic Surfactants, J Surfact Deterg 19(2):363-372.

8. Blagojevic S. M., Pejic N. D., Blagojevic S. N. (2017). Synergism and Physicochemical Properties of Anionic/Amphoteric Surfactant Mixtures with Nonionic Surfactant of Amine Oxide Type, Russian Journal of Physical Chemistry A, 91 (13): 2690-2695.

9. Bouassida M., Fouratil N., Ghazala I., Ellouze-Chaabouni S., Ghribi D. (2018). Potential application of Bacillus subtilis SPB1 biosurfactants in laundry detergent formulations: Compatibility study with detergent ingredients and washing performance, Engineering in Life Sciences, 18 (1): 70-77.

10. Bratovcic A., Nazdrajic S., Odobasic A., Sestan I., The Influence of Type of Surfactant on Physicochemical Properties of Liquid Soap, Int. J. Mater. Chem. 2018, 8(2): 31-37.

11. Chidi O., Adebayo I. V., (2018). Determination of Critical Micelle Concentration and Thermodynamic Evaluations of Micellization of GMS, Mod Chem Appl, 6:2.

12. Cornwel P. A. (2018). A review of shampoo surfactant technology: consumer benefits, raw materials and recent developments, International Journal of Cosmetic Science, 40, 16-30.

13. Czajka A, Hazell G, Eastoe J (2015). Surfactants at the Design Limit, Langmuir, 31, 8205-8217.

14. D. E. Otzen (2017). Biosurfactants and surfactants interacting with membranes and proteins: Same but different? Biochimica et Biophysica Acta (BBA) - Biomembranes, 1859(4): 639-649.

15. El-Shattory Y. A., Elmawla A. G., El-Hamide H. A. A. (2018). Improved Manual Dishwashing Liquid Detergent Compared to that Produced by Multinational Companies in Egyptian Market, Egypt. J. Chem. 61, (4) $651-659$.

16. Fernández-Serrano M., Jurado E., Fernández-Arteaga A., Ríos F., Lechuga M. (2014). Ecotoxicological Assessment of Mixtures of Ether Carboxylic Derivative and Amine-Oxide-Based Non-ionic Surfactants on the Aquatic Environment, J Surfact Deterg, 17: 1161-1168.

17. Garcia M. T., Kaczerewska O., Ribosa I., Brycki B., Materna P., Drgas M. (2016). Biodegradability and aquatic toxicity of quaternary ammonium-based gemini surfactants: effect of the spacer on their ecological properties, Chemosphere, 154, 155-160.

18. Gwiazdowska D. \& Wieczorek D. 2014. Antimicrobial properties of liquid soaps with declared antibacterial effect, Polish Journal of Cosmetology, 17: 78-82.

19. Hassan S., Rehman H. U., Saddique A. (2017). Physiochemical Analysis of Different Soap and Shampoo Collected from the Different Local Market of District Karak, KP, Pakistan, World Applied Sciences Journal, 35 (9) 2012-2014. 
20. Kosmowska N., Łuczak W., Gwiazdowska D., Michocka K., Wieczorek D. (2014) Antibacterial activity of chemical compounds used as surfactants, TOWAROZNAWCZE PROBLEMY JAKOŚCI 2(39): 142 - 149

21. Lavkush Bhaisare M, Pandey S, Shahnawaz Khan M, Talib A, Wu HF. (2015). Fluorophotometric determination of critical micelle concentration (CMC) of ionic and non-ionic surfactants with carbon dots via Stokes shift, Talanta, 132, 572-578.

22. Maurad Z.A., Idris Z., Ghazali R. (2017). Performance of Palm-Based C16/18 Methyl Ester Sulphonate (MES) in Liquid Detergent Formulation, J. Oleo Sci. 66, (7) 677-687.

23. Mendes Cangussú Í., Loiola Vasconcelos T. Y., Feitosa Medeiros D. P., Martins Mesquita R. J., Bezerra Sampaio Marques F. V., de Vasconcelos Saraiva R. L., Ávilo do Nascimento A. (2016). The development of different formulations containing $2 \%$ chlorhexidine digluconate and preliminary evaluation of the stability of the formulations, World Journal of Farmaceutical Research, Volume 5, Issue 5, 139-147.

24. Mitrinova Z., Tcholakova S., Denkov N. (2018). Control of surfactant solution rheology using medium-chain cosurfactants, Colloids and Surfaces A, 537, 173-184.

25. Mortensen HG, Madsen JK, Andersen KK, Vosegaard T, Deen GR, Otzen DE, Pedersen JS (2017). Myoglobin and $\alpha$-Lactalbumin Form Smaller Complexes with the Biosurfactant Rhamnolipid Than with SDS, Biophys J, 113(12):2621-2633.

26. Nguyen K. T., Nguyen A. V., Evans G. M. (2015). Interfacial Water Structure at Surfactant Concentrations below and above the Critical Micelle Concentration as Revealed by Sum Frequency Generation Vibrational Spectroscopy, J. Phys. Chem. C, 119, 15477-15481.

27. Nguyen L. A. T. (2015). Adsorption of non-ionic surfactants onto ultrafiltration membranes in aqueous and organic solutions., Von der Fakultät II -Mathematik und Naturwissenschaftender Technischen Universität Berlinzur Erlangung des akademischen Grades, genehmigte Dissertation, Berlin

28. Odunze U., O’Brien F., Godfrey L., Schätzlein A., Uchegbu Ij. (2019). Unsual enalpy driven self assembly at room temperature with chitosan amphiphiles, Pharm. Nanoteh., 7, 57-71.

29. Osei-Bonsu K., Shokri N., Grassia P. (2015). Foam stability in the presence and absence of hydrocarbons: From bubble- to bulk-scale, Colloids and Surfaces A: Physicochemical and Engineering Aspects, 481: 514-526.

30. Ríos F., Fernández-Arteaga A., Lechuga M., Fernández-Serrano M. (2018) Ecotoxicological Characterization of Surfactants and Mixtures of Them. In: Bidoia E., Montagnolli R. (eds) Toxicity and Biodegradation Testing. Methods in Pharmacology and Toxicology. Humana Press, New York, NY, 311-330.

31. Santos M. S., Tavares F. W., Biscaia Jr E. C. (2016). Molecular thermodynamics of micellization: micelle size distributions and geometry transitions, Braz. J. Chem. Eng. vol. 33 3, 515-523.

32. Sheng Y., Wu X., Lu S., Li C. (2016). Experimental Study on Foam Properties of Mixed Systems of Silicone and Hydrocarbon Surfactants, J Surfact Deterg., 19: 823-831.

33. Tehrani-Bagha A. R., Holmberg K., Ginkel G. C., Kean M. (2015) Cationic gemini surfactants with cleavable spacer: Chemical hydrolysis, biodegradation, and toxicity, J. Colloid Interf. Sci., 431: 72-79.

34. Toofan M. \& Toofan J. (2015). A Brief Review of the Cleaning Process for Electronic Device Fabrication, Developments in Surface Contamination and Cleaning. Wet and Dry Cleaning Methods, 185-2012.

35. Vijayakumar S. \& Saravanan V. (2015). Biosurfactants-Types, Sources and Applications, Research Journal of Microbiology, 10 (5): 181-192.

36. Wieczorek D., Kwaśniewska D., Staszak K., Dobrowolski A. (2017). Effect of amphoteric surfactant on surface and antimicrobial properties of liquid soap, Current Trends in Commodity Science: Cosmetic Products Development, 138-150.

37. Yuan C. L., Xu Z. Z., Fan M. X., Liu H. Y., Xie Y. H., Zhu T., (2014). Study on characteristics and harm of surfactants, J. Chem. Pharm Res. 6(7):2233-2237

38. Zhi L., Li Q., Li Y., Sun Y. (2014). Self-aggregation and Antimicrobial Activity of Saccharide-cationic Surfactants. Colloids and Surfaces A: Physicochemical and Engineering Aspects, 456: 231-237. 